\title{
Participación de las metaloproteinasas de matriz extracelular en la EPOC
}

\author{
Jazmín Hernández-Montoya, ** Gloria Pérez-Rubio, *\$ Julia Pérez Ramos, ${ }^{*}$ \\ Martha Montaño Ramírez,* Carlos Ramos Abraham,* Alejandra Ramírez Venegas,* \\ Ángel Camarena,* Raúl Sansores,* Ramcés Falfán-Valencia*
}

*Instituto Nacional de Enfermedades Respiratorias Ismael Cosío Villegas, Ciudad de México;

*Universidad Autónoma Metropolitana, Unidad Xochimilco;

${ }^{\S}$ Postgrado en Ciencias Biológicas, UNAM.

Trabajo recibido: 17-X-2013; aceptado: 05-XI-2013

RESUMEN. En la enfermedad pulmonar obstructiva crónica (EPOC) se presentan dos fenotipos clínicos, enfisema y bronquitis crónica. Tales fenotipos comparten síntomas clínicos como la disnea y la obstrucción bronquial al flujo de aire, además de un aumento de células de la inmunidad innata (macrófagos y neutrófilos), liberación de múltiples mediadores de la inflamación (quimiocinas, citocinas, factores de crecimiento), estrés oxidativo, aumento de metaloproteinasas de matriz extracelular (MMPs) en las vías aéreas y el pulmón. La progresión de la enfermedad se asocia con la presencia de inflamación crónica y aumento de la actividad proteolítica de enzimas como las MMPs que resulta en la degradación de las fibras de elastina y colágeno en las paredes de los alvéolos y la matriz extracelular del pulmón.

De especial interés para esta revisión ha sido describir la función de las MMPs, su participación en el desarrollo de la EPOC, tanto en la destrucción de la matriz extracelular y la remodelación anormal del pulmón dañado. También se mencionan estudios de asociación genética de polimorfismos tipo SNP (polimorfismo de un solo nucleótido) en MMPs, en poblaciones como la caucásica con el desarrollo y la progresión de la EPOC, además de la importancia de llevar a cabo estudios en poblaciones mestizas. La función de las MMPs también se ha conocido a través de modelos animales como ratones knock-out para MMPs expuestos a humo de cigarro. Todos estos estudios implican a las MMPs como mediadores clave en la patogénesis de la EPOC. Finalmente, el estudio de las MMPs permitirá la base para futuras terapias, posibles blancos terapéuticos y tratamientos de la enfermedad.

Palabras clave: MMPs, EPOC, polimorfismos, SNP.

ABSTRACT. In chronic obstructive pulmonary disease (COPD) are two clinical phenotypes, emphysema and chronic bronchitis. Phenotypes share clinical symptoms such as dyspnea and bronchial obstruction to air flow and an increase of innate immune cells (macrophages and neutrophils), the release of multiple inflammatory mediators (chemokines, cytokines, and growth factors), oxidative stress, increased extracellular matrix metalloproteinases (MMPs) in the airways and lung. Disease progression is associated with the presence of chronic inflammation and increased proteolytic activity of enzymes such as MMPs resulting in the degradation of elastin and collagen fibers in the walls of the alveoli and the lung's extracellular matrix. Of particular interest for this review was to describe the role of MMPs, their participation in the development of COPD, both in the extracellular matrix destruction and abnormal lung remodeling damaged. Also mentioned genetic association studies of polymorphisms in MMPs type SNP in Caucasian populations such as the development and progression of COPD, and the importance of carrying out studies in Mexican mestizo population. The role of MMPs has also been known through animal models and knockout mice MMPs exposed to cigarette smoke. All these studies implicate MMPs as key mediators in the pathogenesis of COPD. Finally, the study of MMPs base allow for future therapies, potential therapeutic targets and disease treatments.

Key words: MMPs, COPD, polymorphism, SNP.

\section{ENFERMEDAD PULMONAR OBSTRUCTIVA CRÓNICA}

La enfermedad pulmonar obstructiva crónica (EPOC) según la iniciativa global para enfermedad pulmonar obstructiva crónica (GOLD 2013, por sus siglas en inglés), ${ }^{1}$ la define como una enfermedad prevenible y tratable, que se caracteriza por presentar limitación del flujo de aire, por lo general es progresiva, se asocia con una respuesta inflamatoria anormal en las vías aéreas; es ocasionada por la exposición prolongada a partículas y gases nocivos, las exacerbaciones y 
comorbilidades contribuyen a la gravedad en algunos pacientes. $^{2}$

Se considera un problema de salud global, la Organización Mundial de la Salud pronostica que para el 2020 será la tercera causa de muerte a nivel mundial, siendo el consumo de cigarro el principal factor de riesgo. ${ }^{3}$ Entre el 15 y $20 \%$ de la población de fumadores desarrolla EPOC. ${ }^{4,5}$ En México, la Secretaría de Salud en 2004 reportó 17,390 muertes asociadas a EPOC secundaria al consumo de tabaco;, ${ }^{6,7}$ a este respecto, la Encuesta Nacional de Adicciones 2011 indica que el $21.7 \%$ de la población entre 12 y 65 años es fumadora activa. ${ }^{8}$

Actualmente la EPOC se define como una enfermedad compleja y multifactorial, donde factores ambientales interactúan con factores genéticos e influyen en la susceptibilidad individual. ${ }^{9}$ Entre los factores genéticos de riesgo se encuentra ampliamente estudiada la deficiencia de $\alpha 1$-antitripsina (AAT),,$^{10}$ la cual genera un desequilibrio entre proteasas y antiproteasas, ocasionando la destrucción de las paredes alveolares. ${ }^{11}$ Se ha comprobado que la AAT es un inhibidor de las proteasas secretado por los neutrófilos durante la inflamación, se sintetiza en el hígado y se encuentra presente en el suero, líquidos tisulares y macrófagos. ${ }^{12}$

La AAT es miembro de la familia de los inhibidores de serín proteasas, los SERPIN (del inglés Serine Proteinase Inhibitors), se encuentran codificados en el gen SERPINA1, el cual posee hasta 100 variantes genéticas de tipo SNP (polimorfimo de un solo nucleótido), pero solo los alelos (PiZ y PiS) se encuentran asociados a la deficiencia de AAT. Sujetos homocigotos con déficit genético del inhibidor de la proteasa AAT tienden a desarrollar enfisema potenciado por fumar cigarros. ${ }^{13}$

Diversos estudios muestran que la EPOC se caracteriza por un incremento a nivel pulmonar de macrófagos alveolares, neutrófilos y linfocitos $T$ citotóxicos, liberación de múltiples mediadores de la inflamación (quimiocinas, citocinas, factores de crecimiento), estrés oxidativo, ${ }^{14}$ aumento de proteasas, catepsinas y metaloproteinasas de matriz extracelular (MMPs). ${ }^{15}$

Algunos pacientes pueden desarrollar destrucción parenquimatosa (enfisema pulmonar) o inflamación bronquial sintomática con tos y expectoración frecuente (bronquitis crónica) de manera variable. La mayoría de los enfermos presentan una mezcla de estos dos tipos de daño, y comparten hallazgos clínicos como la disnea y la obstrucción bronquial al flujo de aire. ${ }^{16}$

El nivel de obstrucción aérea se mide mediante la espirometría, en dicha prueba se obtiene la relación entre el volumen espiratorio forzado en el primer segundo
(FEV ) y la capacidad vital forzada (FVC), que al ser menor de $70 \%$ post-broncodilatador indica obstrucción en la vía aérea, la severidad de dicha obstrucción se evalúa mediante el valor del FEV $_{1}$ ) post-broncodilatador. ${ }^{17,18} \mathrm{Las}$ guías GOLD toman los siguientes valores para asignar la severidad de la enfermedad: $\mathrm{FEV}_{1}>80 \%$ para el grado I (leve), $\mathrm{FEV}_{1} 50-80 \%$ grado II (moderado), $\mathrm{FEV}_{1}$ $30-50 \%$ se asigna al grado III (severo) y $\mathrm{FEV}_{1}<30 \%$ al grado IV (muy severo). ${ }^{1}$

El objetivo de la presente revisión es proporcionar un panorama general de la participación de las MMPs en la patogénesis de la EPOC, comenzando con una descripción sobre la clasificación, función biológica y regulación, así como los modelos animales más empleados, incluye estudios de asociación de las MMPs en EPOC y posibles teorías del papel de las mismas en la patología.

Para la revisión fue empleada la base de datos $\mathrm{NCBI}$ (National Center for Biotechnology Information), ${ }^{19}$ usando las siguientes palabras clave: MMPs, COPD e polymorphisms, inflammation. Se incluyeron artículos entre el año 2007 y 2013.

\section{CLASIFICACIÓN, ESTRUCTURA, FUNCIÓN Y REGULACIÓN DE LAS METALOPROTEINASAS DE MATRIZ EXTRACELULAR}

Las MMPs pertenecen a la superfamilia de las metzincinas, dentro de ésta se encuentran las serralisinas, astacinas y ADAMs/adamalisinas. ${ }^{20,21}$ Son enzimas que degradan componentes de la matriz extracelular (MEC) y participan en el mantenimiento y remodelación de tejidos, dependen de calcio, actúan a pH neutro, se sintetizan como zimógenos en el núcleo y finalmente se almacenan en los gránulos de los macrófagos y neutrófilos. Se presentan asociadas a la membrana plasmática, o bien solubles (figura 1), en el primero de los casos es una proteína transmembranal con 17 dominios y un péptido señal o predominio en el extremo $\mathrm{N}$-terminal necesario para el desplazamiento intracelular del núcleo hasta la membrana, que es eliminado después de la secreción de las metaloproteinasas. ${ }^{23,24}$ Un estudio reciente reportó una isoforma intracelular de la MMP2, que se activa por estrés oxidativo y activa la inmunidad innata. ${ }^{25}$

En el humano se conocen hasta el momento 24 MMPs (tabla 1), con 30 al $50 \%$ de homología entre ellas, han sido clasificadas en subclases según la especificidad del sustrato o componente de la MEC que degradan, ${ }^{26}$ el subgrupo de las colagenasas (MMP1, MMP8, MMP13 y MMP18) son capaces de escindir el colágeno intersticial tipos I, II y III, dando lugar a colágeno desnaturalizado o gelatina; sin embargo, se ha visto 


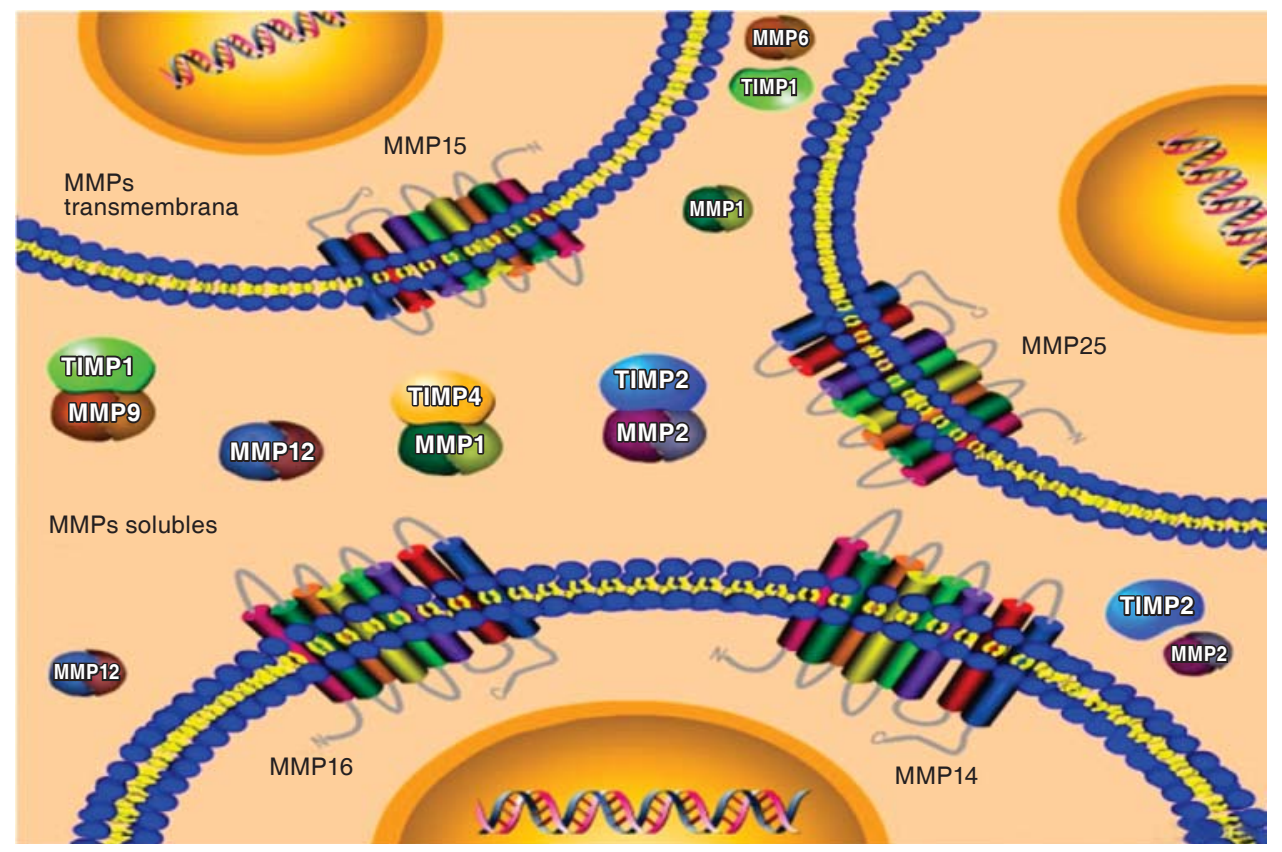

Figura 1.

Esquema representativo de las MMPs descritas actualmente en humano. que también degradan otros componentes de la MEC. El subgrupo de las gelatinasas (MMP2 y MMP9) degrada el colágeno desnaturalizado o gelatina, la MMP2 también degrada colágeno I, II y III, es constitutiva y se encuentra regulada principalmente por activación enzimática y por la estabilidad de su RNA mensajero. ${ }^{27,28}$ El subgrupo de las estromelisinas (MMP3, MMP10 y MMP11) digieren diversos componentes de la MEC. Las matrilisinas (MMP7 y MMP26) degradan proteoglicanos, caseína, elastina, fibronectina, gelatina, vitronectina; y por último, las metaloproteinasas asociadas a membrana, denominadas MT-MMP (del inglés membrane-type matrix metalloproteases), que a su vez se dividen en proteínas transmembrana unidas a la membrana por un sitio hidrófobo (MMP14, MMP15, MMP16, MMP24) y las proteínas que poseen glicofosfatidilinositol (GPI) (MMP17 y MMP25), dichas metaloproteinasas forman parte de las membranas basales e intervienen en la actividad proteolítica de otras MMPs. ${ }^{29}$

Las MMPs desempeñan numerosas funciones en procesos fisiológicos que implican remodelación y mantenimiento tisular, degradación de la MEC activan factores de crecimiento, receptores de superficie y moléculas de adhesión, ${ }^{30}$ morfogénesis tisular, desarrollo embrionario, migración celular, reparación de heridas, desarrollo del hueso; y en procesos patológicos tales como cáncer, enfermedades inflamatorias, autoinmunes, cardiovasculares, EPOC, entre otras. ${ }^{31}$

Bajo condiciones fisiológicas la actividad de las MMPs es regulada a diversos niveles: transcripcional, de zimógenos o por inhibidores endógenos llamados inhibidores de metaloproteinasas tisulares (TIMPs, por sus siglas en inglés), en humanos se han descrito cuatro: TIMP-1, TIMP-2, TIMP-3 y TIMP-4, ${ }^{32}$ actúan a través de su unión directa y reversible al dominio catalítico de las MMPs. ${ }^{33}$ Los inhibidores TIMP-1 y TIMP-2 son secretados en forma soluble, TIMP-3 es asociada con la MEC. ${ }^{34}$ TIMP-1 actúa sobre proMMP-9 y TIMP-2 sobre pro-MMP-2, TIMP-3 inhibe, por lo general, a las adamalisinas, ${ }^{35}$ TIMP-4 ha sido poco estudiada en comparación con los otros tres miembros del grupo, aunque un estudio indica que inhibe a MMP-1 y MMP-2. ${ }^{36}$

Se conoce otro mecanismo de regulación génica de las MMPs, que es la estabilización del ácido ribonucleico mensajero (RNAm) mediante factores de crecimiento, reportes indican que el factor de crecimiento epidérmico (EGF) regula el RNAm de la MMP1 y MMP- $3,{ }^{37}$ mientras que el factor de crecimiento derivado de plaquetas (PDGF) y los glucocorticoides regulan el RNAm de MMP-13. ${ }^{38} \mathrm{El}$ estudio de la regulación de la expresión de las MMPs a los diferentes estímulos extracelulares y como zimógeno latente, es relevante debido a que las MMPs participan en diversos procesos fisiológicos y patológicos que a continuación serán descritos.

\section{PARTICIPACIÓN DE LAS MMPS A NIVEL PULMONAR}

Las principales células del tejido pulmonar que participan en la respuesta inmunológica innata y adaptativa, son los macrófagos, neutrófilos y células epiteliales, 
Tabla 1. Clasificación de las MMPs según el sustrato que degradan.

\begin{tabular}{|c|c|c|}
\hline Clasificación & MMP & Sustrato \\
\hline \multirow[t]{5}{*}{ Colagenasas } & MMP1 & $\begin{array}{l}\text { Colágeno: intersticial, } 1 \text {, de } \\
\text { neutrófilos y } 2\end{array}$ \\
\hline & MMP8 & Colágeno 3 \\
\hline & MMP13 & Colágeno-4 (Xenopus) \\
\hline & MMP18 & RASI-1, RASI-6, MMP19 \\
\hline & MMP19 & Colágeno 1 \\
\hline \multirow[t]{2}{*}{ Gelatinasas } & MMP2 & Gelatina A \\
\hline & MMP9 & Gelatina B, colágeno-2 \\
\hline \multirow[t]{3}{*}{ Estromelisinas } & MMP3 & Estromelisina-1 \\
\hline & MMP10 & Estromelisina-2, transina-2 \\
\hline & MMP11 & Estromelisina 3 \\
\hline \multirow[t]{3}{*}{ Matrilisinas } & MMP7 & Matrilisina, matrina, metalopro- \\
\hline & & $\begin{array}{l}\text { teinasa-1 putativa, metaloendo- } \\
\text { peptidasa uterina }\end{array}$ \\
\hline & MMP26 & Matrilisina 2, endometasa \\
\hline Metaloelastasas & MMP12 & $\begin{array}{l}\text { Elastasa de macrófago, meta- } \\
\text { loeslastasa }\end{array}$ \\
\hline \multirow{8}{*}{$\begin{array}{l}\text { Metaloproteinasas } \\
\text { de matriz }\end{array}$} & MMP14 & MT1-MMP \\
\hline & MMP15 & MT3-MMP \\
\hline & MMP16 & MT3-MMP \\
\hline & MMP17 & MT4-MMP \\
\hline & MMP24 & Metaloproteinasa de matriz tipo \\
\hline & & 5, MT5-MMP \\
\hline & MMP25 & Metaloproteinasa de matriz tipo \\
\hline & & 5, leucolisina, MT6-MMP \\
\hline \multirow[t]{7}{*}{ Otras MMPs } & MMP20 & Amelogenina \\
\hline & MMP21 & Pro-gelatina \\
\hline & MMP22 & Colágeno III, IV, IX, X \\
\hline & MMP23 & No referido \\
\hline & MMP26 & Colágeno tipo IV \\
\hline & MMP27 & MMP22 \\
\hline & MMP28 & Caseína \\
\hline
\end{tabular}

RASI = Sistema de inhibidores renina-angiotensina-aldosterona (del inglés renin-angiotensin-aldosterone system inhibitors.

estas últimas producen MMPs y otros mediadores proinflamatorios. $^{39}$

La actividad enzimática de las metaloproteinasas se relaciona con la remodelación, mantenimiento y reparación de las proteínas de la MEC, principalmente colágeno y elastina,${ }^{40}$ favorecen la migración celular y la formación de vasos sanguíneos mediante el procesamiento de factores angiogénicos, como el factor de crecimiento endotelial vascular (VEGF), factor básico de crecimiento de fibroblastos (bFGF), factor de crecimiento transformante beta (TGF- $\beta$ ) o antiangiogénicos (angiostatina y endostatina). ${ }^{41-45}$ Son capaces de proteolizar diversos receptores celulares para regular la función de los mismos, el receptor activador de plasminógeno de tipo uroquinasa (UPAR), el receptor de TGF- $\beta$ de tipo III, betaglicano o cadherinas del tipo vascular endotelial (VE). ${ }^{46-50}$

En el proceso inflamatorio, la producción de mediadores inflamatorios y MMPs por parte de las células epiteliales constituye un elemento fundamental en el desarrollo del mismo. Se ha demostrado que las células epiteliales de pacientes con EPOC liberan más mediadores proinflamatorios y MMPs que las de fumadores sin EPOC. ${ }^{51}$ La producción aumentada de IL-8, TNF- $\alpha$ y MMPs por parte de las células epiteliales estimula el reclutamiento de otras células inflamatorias (neutrófilos) y amplifica el proceso inflamatorio. ${ }^{52}$

Por otro lado, diversos estudios muestran que las MMPs participan en la formación de tumores y metástasis de varios tipos de cáncer, incluyendo el de pulmón. ${ }^{53}$ Tanto las MMPs como sus inhibidores TIMPs, forman parte de los factores que favorecen la progresión de tumores, ${ }^{54}$ se ha descrito que las MMPs están involucradas en la extravasación de linfocitos a los tejidos, lugar donde cumplen funciones de la respuesta inmunológica, por lo que los linfocitos neoplásicos podrían utilizar este mecanismo en la invasión y metástasis. ${ }^{55,56} \mathrm{En}$ estudios con líneas celulares de melanoma humano, se ha demostrado que la MMP9 se asocia a la membrana celular a través de CD44, confiriendo a las células una mayor capacidad invasiva. ${ }^{57}$

Las MMPs, tales como la MMP-9 y la MMP-12 participan en promover o inhibir la proliferación celular a través del procesamiento de moléculas como el receptor para el factor de crecimiento de fibroblastos 1 (FGR1), el receptor de tirosinas quinasas tipo HER2, o el factor de crecimiento epidérmico de unión a heparina (HB-EGF). ${ }^{58}$

También se ha descrito el papel de las MMPs en la inducción de supervivencia y apoptosis en las células tumorales, a través de la inactivación o liberación del ligando de Fas (FasL) o la ruptura del propio receptor Fas. ${ }^{59}$ Del mismo modo, el proceso de angiogénesis regulado por MMPs resulta fundamental en la progresión tumoral. ${ }^{59}$

En la EPOC la inflamación en el tejido pulmonar desencadena mecanismos de reparación que incluyen la regeneración o formación de tejido nuevo en el que participan las MMPs, principalmente la MMP1, MMP2, MMP9 y MMP $12 .{ }^{60}$ Se ha demostrado que en pacientes con enfisema, en el parénquima pulmonar existe un aumento en la producción de MMP9 y MMP2, dando como resultado la destrucción del mismo y presencia de inflamación. ${ }^{61}$ En otro estudio, también en pacientes con enfisema, se observa un incremento en la expresión de MMP1 y MMP9, comparado con voluntarios sin obstrucción, tal incremento genera destrucción del 
parénquima pulmonar, además de inflamación crónica. ${ }^{62}$ Sin embargo, en otros estudios no se encontraron hallazgos significativos en los niveles de expresión de la MMP12 en pacientes con enfisema, ${ }^{63}$ lo que indica que se requiere de nuevas estrategias para definir la participación de las MMPs en la EPOC.

Los estudios antes mencionados contribuyen al conocimiento de la patología, actualmente, los modelos animales permiten reproducir algunos eventos clave de la EPOC con distintos objetivos, en unos casos dirigidos a estudiar características funcionales o de aplicación de técnicas de diagnóstico, para ensayos terapéuticos experimentales o finalmente, como apoyo para estudios patogénicos de la enfermedad mediante modelos animales expuestos de manera crónica al humo de cigarro.

\section{MODELOS ANIMALES PARA EL ESTUDIO DE LAS MMPS EN EPOC}

El desarrollo de modelos animales es una importante herramienta de trabajo para simular el microambiente pulmonar de la EPOC, es común usar ratones y exponerlos a humo de cigarro, ${ }^{64}$ ya que las ratas han demostrado ser resistentes a desarrollar EPOC..$^{65}$
Algunas de las cepas de ratón que han sido estudiadas en EPOC son NZWLac/J, C57BL6/J, A/J, SJ/L, y $A K R / J$, de las cuales se ha visto que la NZWLac/J es la cepa más resistente a desarrollar EPOC; C57BL6/J, $\mathrm{A} / \mathrm{J}$ y $\mathrm{SJ} / \mathrm{L}$ son medianamente resistentes y la $\mathrm{AKR} / \mathrm{J}$ totalmente susceptible en el desarrollo de la patología ${ }^{66}$ (tabla 2).

Generalmente se emplean ratones deficientes para genes específicos de MMPs, tales deficiencias no son mortales y a simple vista los ratones son muy similares a la cepa silvestre. ${ }^{67}$ Las principales diferencias se encuentran en el proceso de angiogénesis, el desarrollo o remodelamiento del hueso o alteraciones en el sistema inmune y la respuesta inflamatoria, ${ }^{68}$ en los ratones con EPOC se presenta inflamación anormal en el pulmón, infiltración de células proinflamatorias, liberación de citocinas y MMPs (principalmente MMP1, MMP2, MMP9 y MMP12) que generan destrucción del parénquima pulmonar. ${ }^{69}$ Entre los modelos más estudiados se encuentran los ratones knock-out (KO) de TNF- $\alpha$ que se exponen a humo de cigarro, en ellos disminuye la expresión de citocinas, MMP2 y MMP9, además de la infiltración de células inflamatorias. ${ }^{70,71}$ Otro estudio donde emplean ratones transgénicos y $\mathrm{KO}$, reporta la clara participación de las MMPs en el proceso inflama-

Tabla 2. Modelos animales de metaloproteinasas en el desarrollo de EPOC.

\begin{tabular}{|c|c|c|c|}
\hline Cepa de ratón & Fenotipo & Hallazgo & Referencia \\
\hline C57BL/6J & $\begin{array}{l}\text { Enfisema pulmonar por } \\
\text { exposición a humo de cigarro }\end{array}$ & $\begin{array}{l}\text { Incremento de citocinas } \\
\text { proinflamatorias, MMP2, MMP9 y } \\
\text { MMP12 }\end{array}$ & 87 \\
\hline KO de CCR6 & Enfisema pulmonar & $\begin{array}{l}\text { Incremento de la MMP12 que contri- } \\
\text { buye a la inflamación en vías aéreas } \\
\text { y tejido pulmonar }\end{array}$ & 88 \\
\hline KO de PIGF & $\begin{array}{l}\text { Modelo animal que no presenta } \\
\text { enfisema pulmonar }\end{array}$ & $\begin{array}{l}\text { Presencia de enfisema pulmonar } \\
\text { e incremento de TNF- } \alpha \text { y la MMP9 } \\
\text { en ratones KO de PIGF que se les } \\
\text { administró PIGF exógeno }\end{array}$ & 89 \\
\hline $\begin{array}{l}\text { Transgénico que sobreexpresa } \\
\text { MMP1 humana }\end{array}$ & $\begin{array}{l}\text { Disrupción de las paredes alveolares, } \\
\text { coalescencia de los espacios } \\
\text { alveolares, similar a los cambios } \\
\text { morfológicos observados en el } \\
\text { enfisema humano }\end{array}$ & $\begin{array}{l}\text { Sobreexpresión de MMP1 } \\
\text { asociada con EPOC en } \\
\text { ausencia de cáncer de pulmón }\end{array}$ & 90 \\
\hline KO de MMP12 & $\begin{array}{l}\text { Modelo animal que no presenta } \\
\text { enfisema pulmonar }\end{array}$ & $\begin{array}{l}\text { No hay un incremento en los } \\
\text { macrófagos de los pulmones del } \\
\text { ratón KO de la MMP12; por lo tanto, } \\
\text { no desarrolla enfisema en respuesta } \\
\text { a la exposición prolongada de humo } \\
\text { de cigarro }\end{array}$ & 91 \\
\hline
\end{tabular}


torio de EPOC, concluyen que la ausencia de la MMP2, MMP9 y MMP12 en tales ratones expuestos al humo de cigarro no presentan enfisema. ${ }^{72}$

Mediante estos modelos se ha podido establecer que existe regulación de la expresión de las MMPs por citocinas (como el TNF- $\alpha$ ), que a su vez influye en la remodelación y el rediseño del parénquima pulmonar cuando se pierde la homeostasis de las MMPs y los TIMPs por exposición a partículas del humo de cigarro. ${ }^{73}$ Otro modelo animal en el que se indujo ausencia de la expresión del receptor de citocinas CCR6, la capacidad de desarrollar enfisema en respuesta al humo de cigarro queda limitada, debido a una menor respuesta inflamatoria y disminución de la presencia de proteasas y MMP1, MMP2, MMP9 y MMP12, hecho que permite concluir que la interacción del receptor CCR6 con su ligando y las MMPs participan en la patogénesis de la EPOC, generando principalmente un fenotipo de enfisema pulmonar. ${ }^{74}$

Otra vía empleada para generar enfisema es la administración repetida de endotoxinas bacterianas, lipopolisacáridos (LPS) por vía endotraqueal, en el pulmón de los ratones se observa reclutamiento de neutrófilos y la activación de macrófagos, se produce una respuesta inflamatoria y activación de mediadores, como TNF- $\alpha$, IL-1, IL-8, MMP1, MMP2, MMP9 y MMP12. ${ }^{75}$ En un estudio de expresión mediante microarreglos en ratones de la cepa AKR/J que previamente fueron expuestos al humo de cigarro y LPS bacteriano, encontraron sobreexpresión de la MMP12 al compararlos con la cepa silvestre. ${ }^{76}$ Por otro lado, se ha evaluado la administración del antagonista específico del receptor de citocinas CXCR2 (SB-332235) encontrando que se reduce la respuesta inflamatoria porque disminuye el incremento de neutrófilos y liberación de citocinas y MMPs. ${ }^{77}$
Cada uno de los modelos animales empleados en EPOC permite establecer la participación de las MMPs en la patología, así como los posibles mecanismos por los cuales pueden ser reguladas. Lo anterior propone nuevas hipótesis que será necesario probarlas y validarlas para ser usados como posibles blancos terapéuticos.

\section{POLIMORFISMOS GENÉTICOS DE MMPS Y SU ASOCIACIÓN CON EPOC}

Como se ha mencionado en secciones anteriores, en la EPOC participa cierto componente genético; en el humano se han identificado variaciones genéticas en el DNA conocidas como polimorfismos. Existen varios tipos de polimorfismos (inserciones, deleciones, cambios en el número de secuencias repetidas, etc.), pero los más frecuentes son los SNP, la mayoría de los genes de las MMPs tienen cientos de éstos ${ }^{78} y$ algunos han sido asociados a EPOC (tabla 3). Al menos 20 polimorfismos en el gen MMP1 han sido descritos asociados con la EPOC, ${ }^{79}$ principalmente en regiones reguladoras (promotor, 3'UTR, 5'UTR e intrones). ${ }^{80}$ Algunos estudios de asociación genética de las MMPs con la EPOC son: el polimorfismo (1G-1607/2G), de inserción/deleción en la región promotora del gen MMP1, el cual aumenta la transcripción puesto que introduce un nuevo sitio de unión para el factor de transcripción ETS- ${ }^{81}$ y se asocia a un decline de la función pulmonar de los fumadores. ${ }^{82}$ Otro hallazgo es el polimorfismo rs243865 (C-1306T) del gen $M M P 2$, que se sitúa en el intrón y la variante del alelo T presenta una asociación significativa con el decline del $\mathrm{FEV}_{1}{ }^{83}$ El polimorfismo rs13925 (C-1562T) del gen MMP9 ubicado en el promotor se asocia con el desarrollo de EPOC en población japonesa ${ }^{84}$ y china. ${ }^{85}$

El polimorfismo rs2276109 (A82G), del gen MMP12, se asocia con un incremento en la actividad del pro-

Tabla 3. Estudios de asociación genética de polimorfismos tipo SNPs de MMPs en EPOC.

\begin{tabular}{|c|c|c|c|c|c|c|c|}
\hline Gen & $\mathrm{Cr}$ & Polimorfismo & Valor de $p$ & OR (IC 95\%) & Hallazgo & Población & $\begin{array}{l}\text { Refe- } \\
\text { rencia }\end{array}$ \\
\hline MMP12 & 11 & $\begin{array}{l}\text { rs } 2276109 \\
\text { A-82G }\end{array}$ & 0.02 & $(0.46-0.92)$ & $\begin{array}{l}\text { El alelo G se encuentra } \\
\text { asociado con } \mathrm{FEV}_{1} \text { en asma } \\
\text { y EPOC }\end{array}$ & Caucásica & 92 \\
\hline MMP1 & 11 & $\begin{array}{l}\text { G-1607GG } \\
\text { inserción / } \\
\text { deleción }\end{array}$ & 0.02 & No referido & $\begin{array}{l}\text { El polimorfismo G-1607GG se } \\
\text { asocia con un decline de la } \\
\text { función pulmonar. }\end{array}$ & Caucásica & 93 \\
\hline MMP2 & 16 & $\begin{array}{l}\text { rs } 243865 \\
\text { C-1306T }\end{array}$ & 0.03 & No referido & $\begin{array}{l}\text { Genotipo TT asociado con un } \\
\text { decline en FEV }\end{array}$ & Caucásica & 94 \\
\hline MMP9 & 20 & $\begin{array}{l}\text { rs } 3918242 \\
(-1562 \mathrm{C} / \mathrm{T})\end{array}$ & 0.01 & $0.08(0.01-0.62)$ & $\begin{array}{l}\text { Asociación con EPOC de la } \\
\text { variante del alelo C }\end{array}$ & Caucásica & 95 \\
\hline
\end{tabular}


motor y tiene efecto en el elemento cis del factor de transcripción AP-1, ${ }^{86}$ además se encuentra asociado con la severidad de la EPOC. ${ }^{86}$

Los polimorfismos descritos contribuyen a la descripción de la patogénesis de la EPOC y proporcionan información sobre las bases moleculares de la misma, estos hallazgos han sido reportados principalmente en población caucásica, resulta necesario validar dichos hallazgos en poblaciones mestizas, en las que no existen reportes de asociación de las MMPs con la EPOC.

\section{CONCLUSIONES}

La EPOC es una enfermedad compleja y multifactorial, existe un proceso inflamatorio crónico en las vías aéreas, se desarrolla de manera progresiva y tiene manifestaciones clínicas como enfisema y bronquitis.

El proceso inflamatorio a nivel molecular es generado por la acción y regulación de varias citocinas proinflamatorias y MMPs; éstas últimas producidas por macrófagos, neutrófilos y células epiteliales. Son proteínas capaces de rediseñar y remodelar el parénquima pulmonar, participar como moléculas de señalización en mecanismos como la apoptosis y el ciclo celular, se expresan en respuesta a diferentes estímulos como el estrés oxidativo, generando una regulación compleja en el proceso inflamatorio subyacente.

Las principales MMPs que participan en el proceso inflamatorio, destrucción del parénquima pulmonar y que en estudios de asociación genética se perfilan como genes candidato asociados a la EPOC son: MMP1, MMP2, MMP9y MMP12, algunos estudios clínicos correlacionan los niveles de las respectivas MMPs en suero y plasma con los genotipos obtenidos en los pacientes. El conocimiento generado ha sido importante para describir la patogénesis, desarrollo y progresión de la EPOC por parte de las MMPs.

\section{REFERENCIAS}

1. Global Initiative for Chronic Obstructive Lung Disease (GOLD). Global Strategy for the Diagnosis, Management, and Prevention of Chronic Obstructive Pulmonary Disease NHLBI/WHO Workshop report. Rev. ed. 2013. Access date: 2013 June 3. Available from: http: //www.goldcopd. com

2. Celli BR, MacNee W; ATS/ERS Task Force. Standards for the diagnosis and treatment of patients with COPD: a summary of the ATS/ERS position paper. Eur Respir J 2004;23(6):932-946.

3. Organización Mundial de la Salud (OMS). Fecha de acceso: junio 3, 2013. Disponible en: http://www.who.int/es/
4. Barnes PJ. Mediators of chronic obstructive pulmonary disease. Pharmacol Rev 2004;56(4):515-548.

5. Barnes PJ. Chronic obstructive pulmonary disease: a growing but neglected global epidemic. PLoS Med 2007;4(5):e112.

6. Secretaría de Salud. Fecha de acceso: junio 3, 2013. Disponible en: http://www.salud.gob.mx/

7. Hogg JC, Chu F, Utokaparch S, et al. The nature of smallairway obstruction in chronic obstructive pulmonary disease. N Engl J Med 2004;350(26):2645-2653.

8. Encuesta Nacional de Adicciones 2011. Fecha de acceso: junio 3, 2013. Disponible en: http://www.conadic.salud. gob.mx/pdfs/ENA_2011_DROGAS_ILICITAS_.pdf

9. Silverman EK, Sandhaus RA. Clinical practice. Alpha1-antitrypsin deficiency. N Engl J Med 2009;360(26):2749-2757.

10. Strange C, Stoller JK, Sandhaus RA, Dickson R, Turino G. Results of a survey of patients with alpha-1 antitrypsin deficiency. Respiration 2006;73(2):185-190.

11. Stockley RA. Neutrophils and protease/antiprotease imbalance. Am J Respir Crit Care Med 1999;160(5 Pt 2):S49-S52.

12. Silverman EK, Chapman HA, Drazen JM, et al. Genetic epidemiology of severe, early-onset chronic obstructive pulmonary disease. Risk to relatives for airflow obstruction and chronic bronchitis. Am J Respir Crit Care Med 1998; 157(6 Pt 1):1770-1778.

13. Brusselle GG, Joos GF, Bracke KR. New insights into the immunology of chronic obstructive pulmonary disease. Lancet 2011;378(9795):1015-1026.

14. Mahadeva R, Shapiro SD. Chronic obstructive pulmonary disease * 3: Experimental animal models of pulmonary emphysema. Thorax 2002;57(10):908-914.

15. Mannino DM. Chronic obstructive pulmonary disease: definition and epidemiology. Respir Care 2003;48(12):1185-1191.

16. Pauwels RA, Buist AS, Calverley PM, Jenkins $C R$, Hurd SS; GOLD Scientific Committee. Global strategy for the diagnosis, management, and prevention of chronic obstructive pulmonary disease. NHLBI/WHO Global Initiative for Chronic Obstructive Lung Disease (GOLD) Workshop summary. Am J Respir Crit Care Med 2001;163(5):1256-1276.

17. Raherison C, Girodet PO. Epidemiology of COPD. Eur Respir Rev 2009;18(114):213-221.

18. National Center for Biotechnology Information (NCBI). Access date: 2013 June 3. Available from: http://www. ncbi.nlm.nih.gov/

19. Bode W, Grams F, Reinemer P, et al. The metzincinsuperfamily of zinc-peptidases. Adv Exp Med Biol 1996;389:1-11.

20. Stocker W, Bode W. Structural features of a superfamily of zinc-endopeptidases: the metzincins. Curr Opin Struct Biol 1995;5(3):383-390.

21. Nagase $H$, Visse R, Murphy G. Structure and function of matrix metalloproteinases and TIMPs. Cardiovasc Res 2006 15;69(3):562-573. 
22. Pathway Builder Tool 2.0 available from Protein Lounge, San Diego, CA, USA. Fecha de acceso: 3 de junio, 2013.

23. Maskos K. Crystal structures of MMPs in complex with physiological and pharmacological inhibitors. Biochimie 2005;87(3-4):249-263.

24. Lovett $\mathrm{DH}$, Mahimkar R, Raffai RL, et al. A novel intracellular isoform of matrix metalloproteinase-2 induced by oxidative stress activates innate immunity. PLoS One 2012;7(4):e34177.

25. Sternlicht MD, Werb Z. How matrix metalloproteinases regulate cell behavior. Annu Rev Cell Dev Biol 2001;17:463-516.

26. Overall $\mathrm{CM}$, Wrana JL, Sodek J. Transcriptional and post-transcriptional regulation of $72-k D a$ gelatinase/ type IV collagenase by transforming growth factor-beta 1 in human fibroblasts. Comparisons with collagenase and tissue inhibitor of matrix metalloproteinase gene expression. J Biol Chem 1991;266(21):14064-14071.

27. Strongin AY, Collier I, Bannikov G, Marmer BL, Grant GA, Goldberg GI. Mechanism of cell surface activation of 72-kDa type IV collagenase. Isolation of the activated form of the membrane metalloprotease. J Biol Chem 1995;270(10):5331-5338.

28. Seiki M. Membrane-type matrix metalloproteinases. APMIS 1999;107(1):137-143.

29. Cauwe B, Martens E, Van den Steen PE, et al. Adenylyl cyclase-associated protein-1/CAP1 as a biological target substrate of gelatinase B/MMP-9. Exp Cell Res 2008;314(15):2739-2749.

30. Egeblad M, Werb Z. New functions for the matrix metalloproteinases in cancer progression. Nat Rev Cancer 2002;2(3):161-174.

31. Brew K, Dinakarpandian D, Nagase H. Tissue inhibitors of metalloproteinases: evolution, structure and function. Biochim Biophys Acta 2000;1477(1-2):267-283.

32. Löffek S, Schilling O, Franzke CW. Series "matrix metalloproteinases in lung health and disease": Biological role of matrix metalloproteinases: a critical balance. Eur Respir J 2011;38(1):191-208.

33. Lagente $V$, Manoury $B$, Nénan S, Le Quément $C$, MartinChouly C, Boichot E. Role of matrix metalloproteinases in the development of airway inflammation and remodeling. Braz J Med Biol Res 2005;38(10):1521-1530.

34. Moore CS, Crocker SJ. An alternate perspective on the roles of TIMPs and MMPs in pathology. Am J Pathol 2012;180(1):12-16.

35. Bourboulia D, Stetler-Stevenson WG. Matrix metalloproteinases (MMPs) and tissue inhibitors of metalloproteinases (TIMPs): Positive and negative regulators in tumor cell adhesion. Semin Cancer Biol 2010;20(3):161-168.

36. Baker AH, Edwards DR, Murphy G. Metalloproteinase inhibitors: biological actions and therapeutic opportunities. J Cell Sci 2002;115(Pt 19):3719-3727.

37. Delany AM, Jeffrey JJ, Rydziel S, Canalis E. Cortisol increases interstitial collagenase expression in osteoblasts by post-transcriptional mechanisms. J Biol Chem 1995;270(44):26607-26612.
38. Pendás AM, Balbín M, Llano E, Jiménez MG, López-Otín C. Structural analysis and promoter characterization of the human collagenase-3 gene (MMP13). Genomics 1997;40(2):222-233.

39. Page-McCaw A, Ewald AJ, Werb Z. Matrix metalloproteinases and the regulation of tissue remodelling. Nat Rev Mol Cell Biol 2007;8(3):221-233.

40. Imai K, Dalal SS, Chen ES, et al. Human collagenase (matrix metalloproteinase-1) expression in the lungs of patients with emphysema. Am J Respir Crit Care Med 2001;163(3 Pt 1):786-791.

41. Brinckerhoff CE, Matrisian LM. Matrix metalloproteinases: a tail of a frog that became a prince. Nat Rev Mol Cell Biol 2002;3(3):207-314.

42. Mira E, Lacalle RA, Buesa JM, et al. Secreted MMP9 promotes angiogenesis more efficiently than constitutive active MMP9 bound to the tumor cell surface. J Cell Sci 2004;117(Pt 9):1847-1857.

43. Stamenkovic I. Extracellular matrix remodelling: the role of matrix metalloproteinases. J Pathol 2003;200(4):448-464.

44. Zanini A, Chetta A, Imperatori AS, Spanevello A, Olivieri $D$. The role of the bronchial microvasculature in the airway remodelling in asthma and COPD. Respir Res 2010;11:132.

45. Nagira M, Imai T, Yoshida R, et al. A lymphocyte-specific CC chemokine, secondary lymphoid tissue chemokine (SLC), is a highly efficient chemoattractant for B cells and activated $T$ cells. Eur J Immunol 1998;28(5):1516-1523.

46. Luca M, Huang S, Gershenwald JE, Singh RK, Reich $\mathrm{R}$, Bar-Eli M. Expression of interleukin-8 by human melanoma cells up-regulates MMP-2 activity and increases tumor growth and metastasis. Am J Pathol 1997;151(4):1105-1113.

47. Alejandre-Alcázar MA, Michiels-Corsten M, Vicencio AG, et al. TGF-beta signaling is dynamically regulated during the alveolarization of rodent and human lungs. Dev Dyn 2008;237(1):259-269.

48. Wojtowicz-Praga SM, Dickson RB, Hawkins MJ. Matrix metalloproteinase inhibitors. Invest New Drugs 1997;15(1):61-75.

49. Yu Q, Stamenkovic I. Cell surface-localized matrix metalloproteinase-9 proteolytically activates TGF-beta and promotes tumor invasion and angiogenesis. Genes Dev 2000;14(2):163-176.

50. Vignola AM, Paganin F, Capieu L, et al. Airway remodelling assessed by sputum and high-resolution computed tomography in asthma and COPD. Eur Respir J 2004;24(6):910-917.

51. Culpitt SV, de Matos C, Russell RE, Donnelly LE, Rogers DF, Barnes PJ. Effect of theophylline on induced sputum inflammatory indices and neutrophil chemotaxis in chronic obstructive pulmonary disease. Am J Respir Crit Care Med 2002;165(10):1371-1376.

52. Deryugina El, Quigley JP. Matrix metalloproteinases and tumor metastasis. Cancer Metastasis Rev 2006;25(1):9-34.

53. Houghton AM, Grisolano JL, Baumann ML, et al. Macrophage elastase (matrix metalloproteinase-12) suppresses growth of lung metastases. Cancer Res 2006;66(12):6149-6155. 
54. Lambert $E$, Dassé $E$, Haye $B$, Petitfrère $E$. TIMPs as multifacial proteins. Crit Rev Oncol Hematol 2004;49(3):187-198.

55. Leppert D, Waubant E, Galardy R, Bunnett NW, Hauser SL. T cell gelatinases mediate basement membrane transmigration in vitro. J Immunol 1995;154(9):4379-4389.

56. Haas TL, Madri JA. Extracellular matrix-driven matrix metalloproteinase production in endothelial cells: implications for angiogenesis. Trends Cardiovasc Med 1999;9(3-4):70-77.

57. Yu Q, Stamenkovic I. Localization of matrix metalloproteinase 9 to the cell surface provides a mechanism for CD44-mediated tumor invasion. Genes Dev 1999;13(1):35-48.

58. Suzuki M, Raab G, Moses MA, Fernandez CA, Klagsbrun M. Matrix metalloproteinase-3 releases active heparin-binding EGF-like growth factor by cleavage at a specific juxtamembrane site. J Biol Chem 1997;272(50):31730-31737.

59. Holmbeck K, Bianco P, Chrysovergis K, Yamada S, Birkedal-Hansen H. MT1-MMP-dependent, apoptotic remodeling of unmineralized cartilage: a critical process in skeletal growth. J Cell Biol 2003;163(3):661-671.

60. Ohnishi K, Takagi M, Kurokawa Y, Satomi S, Konttinen YT. Matrix metalloproteinase-mediated extracellular matrix protein degradation in human pulmonary emphysema. Lab Invest 1998;78(9):1077-1087.

61. Finlay GA, O’Driscoll LR, Russell KJ, et al. Matrix metaIloproteinase expression and production by alveolar macrophages in emphysema. Am J Respir Crit Care Med 1997;156(1):240-247.

62. Lim S1, Roche N, Oliver BG, Mattos W, Barnes PJ, Chung KF. Balance of matrix metalloprotease-9 and tissue inhibitor of metalloprotease-1 from alveolar macrophages in cigarette smokers. Regulation by interleukin-10. Am J Respir Crit Care Med 2000;162(4 Pt 1):1355-1360.

63. Shapiro SD. Transgenic and gene-targeted mice as models for chronic obstructive pulmonary disease. Eur Respir J 2007;29(2):375-378.

64. Shapiro SD. Proteinases in chronic obstructive pulmonary disease. Biochem Soc Trans 2002;30(2):98-102.

65. Guerassimov A, Hoshino Y, Takubo Y, et al. The development of emphysema in cigarette smoke-exposed mice is strain dependent. Am J Respir Crit Care Med 2004;170(9):974-980.

66. Reynolds PR, Cosio MG, Hoidal JR. Cigarette smokeinduced Egr-1 upregulates proinflammatory cytokines in pulmonary epithelial cells. Am J Respir Cell Mol Biol 2006;35(3):314-319.

67. Goldklang MP, Marks SM, D'Armiento JM. Second hand smoke and COPD: lessons from animal studies. Front Physiol 2013;4:30.

68. Roycik MD, Fang X, Sang QX. A fresh prospect of extracellular matrix hydrolytic enzymes and their substrates. Curr Pharm Des 2009;15(12):1295-1308.

69. Wright JL, Farmer SG, Churg A. Synthetic serine elastase inhibitor reduces cigarette smoke-induced emphysema in guinea pigs. Am J Respir Crit Care Med 2002;166(7):954-960.

70. Ismair MG1, Ries C, Lottspeich F, Zang C, Kolb HJ, Petrides PE. Autocrine regulation of matrix metallo- proteinase-9 gene expression and secretion by tumor necrosis factor-alpha (TNF-alpha) in NB4 leukemic cells: specific involvement of TNF receptor type 1. Leukemia 1998;12(7):1136-1143.

71. Churg A, Cosio M, Wright JL. Mechanisms of cigarette smoke-induced COPD: insights from animal models. Am J Physiol Lung Cell Mol Physiol 2008;294(4):L612-631.

72. Wright $\mathrm{J}$, Tai $\mathrm{H}$, Churg A. Vasoactive mediators and pulmonary hypertension after cigarette smoke exposure in the guinea pig. J Appl Physiol (1985). 2006;100(2):672-678.

73. Wright JL, Cosio M, Churg A. Animal models of chronic obstructive pulmonary disease. Am J Physiol Lung Cell Mol Physiol 2008;295(1):L1-L15.

74. Spond J, Chapman R, Fine J, et al. Comparison of PDE 4 inhibitors, rolipram and SB 207499 (ariflo), in a rat model of pulmonary neutrophilia. Pulm Pharmacol Ther 2001;14(2):157-164.

75. Jin SL, Conti M. Induction of the cyclic nucleotide phosphodiesterase PDE4B is essential for LPS-activated TNF-alpha responses. Proc Natl Acad Sci USA 2002;99(11):7628-7633.

76. Stevenson CS, Coote K, Webster R, et al. Characterization of cigarette smoke-induced inflammatory and mucus hypersecretory changes in rat lung and the role of CXCR2 ligands in mediating this effect. Am J Physiol Lung Cell Mol Physiol 2005;288(3):L514-L522.

77. Shimajiri S, Arima N, Tanimoto A, et al. Shortened microsatellite d(CA)21 sequence down-regulates promoter activity of matrix metalloproteinase 9 gene. FEBS Lett 1999;455(1-2):70-74.

78. Vlaykova T, Dimov D. Polymorphisms of matrix metalloproteinases (MMP) in COPD. Biotechnol \& Biotechnol Eq 2012;26(1):111-120.

79. Haq I, Chappell S, Johnson SR, et al. Association of MMP2 polymorphisms with severe and very severe COPD: a case control study of MMPs-1, 9 and 12 in a European population. BMC Med Genet 2010;11:7.

80. Rutter JL, Mitchell TI, Butticè G, et al. A single nucleotide polymorphism in the matrix metalloproteinase-1 promoter creates an Ets binding site and augments transcription. Cancer Res 1998;58(23):5321-5325.

81. Affara M, Dunmore BJ, Sanders DA, Johnson N, Print CG, Charnock-Jones DS. MMP1 bimodal expression and differential response to inflammatory mediators is linked to promoter polymorphisms. BMC Genomics 2011;12:43.

82. Wallace AM, Mercer BA, He J, et al. Functional characterization of the matrix metalloproteinase-1 cigarette smoke-responsive region and association with the lung health study. Respir Res 2012;13:79.

83. Raza SL, Nehring LC, Shapiro SD, Cornelius LA. Proteinase-activated receptor-1 regulation of macrophage elastase (MMP-12) secretion by serine proteinases. J Biol Chem 2000;275(52):41243-41250.

84. Minematsu N, Nakamura H, Tateno H, Nakajima T, Yamaguchi K. Genetic polymorphism in matrix metaIloproteinase-9 and pulmonary emphysema. Biochem Biophys Res Commun 2001;289(1):116-119. 
85. Zhou M, Huang SG, Wan HY, Li B, Deng WW, Li M. Genetic polymorphism in matrix metalloproteinase-9 and the susceptibility to chronic obstructive pulmonary disease in Han population of south China. Chin Med J (Engl) 2004;117(10):1481-1484.

86. Jormsjö S, Ye S, Moritz J, et al. Allele-specific regulation of matrix metalloproteinase-12 gene activity is associated with coronary artery luminal dimensions in diabetic patients with manifest coronary artery disease. Circ Res 2000;86(9):998-1003.

87. Vecchio D, Arezzini B, Pecorelli A, Valacchi G, Martorana PA, Gardi C. Reactivity of mouse alveolar macrophages to cigarette smoke is strain dependent. Am J Physiol Lung Cell Mol Physiol 2010;298(5):L704-L713.

88. Bracke KR, D'hulst Al, Maes T, et al. Cigarette smokeinduced pulmonary inflammation and emphysema are attenuated in CCR6-deficient mice. J Immunol 2006;177(7):4350-4359.

89. Cheng SL, Wang HC, Yu CJ, et al. Prevention of elastaseinduced emphysema in placenta growth factor knockout mice. Respir Res 2009;10:115.

90. D'Armiento J, Dalal SS, Okada Y, Berg RA, Chada K. Collagenase expression in the lungs of transgenic mice causes pulmonary emphysema. Cell 1992;71(6):955-961.

91. Hautamaki RD, Kobayashi DK, Senior RM, Shapiro SD. Requirement for macrophage elastase for ciga- rette smoke-induced emphysema in mice. Science 1997;277(5334):2002-2004.

92. Hunninghake $\mathrm{GM}$, Cho $\mathrm{MH}$, Tesfaigzi $\mathrm{Y}$, et al. MMP12, lung function, and COPD in high-risk populations. N Engl J Med 2009;361(27):2599-2608.

93. Joos L, He JQ, Shepherdson MB, et al. The role of matrix metalloproteinase polymorphisms in the rate of decline in lung function. Hum Mol Genet 2002;11(5):569-576.

94. van Diemen CC1, Postma DS, Siedlinski M, Blokstra A, Smit HA, Boezen HM. Genetic variation in TIMP1 but not MMPs predict excess FEV1 decline in two general population-based cohorts. Respir Res 2011;12:57.

95. Lee SY, Kim MJ, Kang HG, et al. Polymorphisms in matrix metalloproteinase-1, -9 and -12 genes and the risk of chronic obstructive pulmonary disease in a Korean population. Respiration 2010;80(2):133-138.

\section{$\triangle$ Correspondencia:}

Dr. Ramcés Falfán-Valencia, Instituto Nacional de Enfermedades Respiratorias Ismael Cosío Villegas. Calzada de Tlalpan 4502, Colonia Sección XVI. México, D.F., 14080

Correo electrónico: rfalfanv@iner.gob.mx

Los autores declaran no tener conflicto de intereses. 